\title{
Clinicopathological and Molecular Genomic Features of Monomorphic Epitheliotropic Intestinal T-Cell Lymphoma in The Chinese Population: A Study of 20 Cases
}

\section{Chunni Chen}

Jiangsu Province Hospital and Nanjing Medical University First Affiliated Hospital

\section{Yuxi Gong}

Jiangsu Province Hospital and Nanjing Medical University First Affiliated Hospital

\section{Yefan Yang}

Jiangsu Province Hospital and Nanjing Medical University First Affiliated Hospital

\section{Qiuyuan Xia}

Nanjing Jinling Hospital: East Region Military Command General Hospital

\section{Qiu Rao}

Nanjing Jinling Hospital: East Region Military Command General Hospital

\section{Yang Shao}

Nanjing Geneseeq Technology Inc.

\section{Liuqing Zhu}

Nanjing Geneseeq Technology Inc.

\section{Junli Zhang}

Nanjing Geneseeq Technology Inc.

\section{Xiao Li}

Jiangsu Province Hospital and Nanjing Medical University First Affiliated Hospital

\section{Pan Ji}

Jiangsu Province Hospital and Nanjing Medical University First Affiliated Hospital

\section{Boya Zhai}

Jiangsu Province Hospital and Nanjing Medical University First Affiliated Hospital

\section{Xiang Zhang}

Jiangsu Province Hospital and Nanjing Medical University First Affiliated Hospital

Zhihong Zhang ( $\square$ zhangzh@njmu.edu.cn )

Jiangsu Province Hospital and Nanjing Medical University First Affiliated Hospital https://orcid.org/0000-0002-5779-9365

\section{Research}


Keywords: monomorphic epitheliotropic intestinal T-cell lymphoma, whole-genome sequencing, JAK-STAT pathway, amplification of chromosome 9q, ZDBF2

Posted Date: September 7th, 2021

DOI: https://doi.org/10.21203/rs.3.rs-798886/v1

License: (1) This work is licensed under a Creative Commons Attribution 4.0 International License. Read Full License

Version of Record: A version of this preprint was published at Diagnostic Pathology on December 1st, 2021. See the published version at https://doi.org/10.1186/s13000-021-01173-5. 


\section{Abstract \\ Background}

Monomorphic epitheliotropic T-cell lymphoma (MEITL) is an aggressive non-Hodgkin lymphoma with a high fatality rate. This study was aimed to explore the clinicopathological and molecular genetic features of MEITL in the Chinese population.

\section{Methods}

A retrospective analysis was performed based on the clinical manifestations and pathological features of 20 Chinese MEITL. 9 cases with paired diseased-normal tissues were also analyzed for molecular information by whole-genome sequencing.

\section{Results}

There were 14 men and 6 women with a median age of $62.5(28-81)$ years. $17(17 / 20)$ lesions were located in the jejunum or ileum; 13(13/20) cases had ulcers or perforations. Microscopically, except for $1(1 / 20)$ case of pleomorphic cells, the monomorphic, middle-sized tumor cells infiltrating into the intestinal epithelial and peripheral intestinal mucosa recess could be seen in the other 19 cases. Immunohistochemistry showed that most of the tumor cells in MEITL were positive for CD3(20/20), CD8(17/20), CD43(19/20), and CD56(15/20), but negative for CD5(20/20). The most frequently mutated genes of these Chinese cases were STAT5B (4/9) and TP53 (4/9), not SETD2(2/9). ZDBF2 mutations $(4 / 9)$ were also detected for the first time with a high mutated frequency. We demonstrated that mutations of JAK-STAT pathway-related genes and the amplification of Chromosome $9 q$ appeared at the same time in most cases(5/9).

\section{Conclusions}

The clinicopathological features were consistent with that in previous western studies, but a novel mutated gene, ZDBF2, was found in this study. A combination of gene mutations involved in the JAKSTAT pathway and the amplification of Chr9q may be used as one of the molecular features of MEITL diagnosis, especially when morphological and immunohistochemical evidence is insufficient.

\section{Introduction}

Monomorphic epitheliotropic intestinal T-cell lymphoma (MEITL) is an aggressive intestinal non-Hodgkin lymphoma originating from $T$ cells, which has been classified as a separate category from enteropathyassociated T-cell lymphoma (EATL) in the 2016 WHO classification of hematopoietic and lymphoid tissues[1]. MEITL is more common in Asia with monomorphic CD3 + CD8 + CD56 + tumor cells and lacks 
clinical evidence of celiac sprue[2]. Frequent mutations in SETD2, which encodes a non-redundant H3K36specific trimethyltransferase, have been found in most MEITL cases in several studies, and the JAK/STAT pathway is also reported to be the most commonly mutated signaling pathway[3-5].

Due to its low incidence, and a lack of researches on large-scale cases, the clinicopathological and molecular characteristics of MEITL in the Chinese population have not been fully clarified. The present study performed a retrospective analysis based on clinicopathological features of 20 MEITL cases, nine of which also underwent whole-genome sequencing for molecular genetic information, to provide a basis for clinical diagnosis and treatment and supplement the genetic landscape of MEITL in the Chinese population.

\section{Materials And Methods Sample selection}

The use of samples in this study was in accordance with the Declaration of Helsinki and approved by the Ethics Committee of The First Affiliated Hospital of Nanjing Medical University (2020-SR-169).

Patients were eligible for study inclusion if they: 1) were comprehensively diagnosed with MEITL based on pathological morphology, imaging examination, serological indicators, and clinical manifestations. 2) had complete clinical data. 3) had undergone surgical resection between 2013 and 2019.

Fifteen MEITL cases from The First Affiliated Hospital of Nanjing Medical University and 5 MEITL cases from Nanjing Jinling Hospital met the inclusion criteria. Hematoxylin-eosin staining and immunohistochemical slides were retrieved and re-evaluated by two pathologists (Z. Zhang and C. Chen). Nine cases with enough paired diseased-normal tissues were selected for whole-genome sequencing.

Patients' general information, clinical manifestations, pathological morphology, immunohistochemical results, and molecular genetic features were collected for subsequent analysis.

\section{Immunohistochemistry}

Immunohistochemical staining was performed on $3 \mu \mathrm{m}$ thick formalin-fixed paraffin-embedded tissue sections using Ventana automated (BenchMarker) stainer (Tucson, Roche, USA) according to the manufacturer's instructions. Standard immunohistochemical studies were performed using the following antibodies: CD3, CD5, CD8, CD43, CD56, TIA-1, Granzyme B, perforin, Ki-67. All antibodies and EBER probes were ready to use and bought from Maxim biotechnology development co. LTD (China).

Immunohistochemistry results were scored according to the intensity of staining and the percentage of positive cells. The IHC results of all cases were interpreted by two pathologists using a double-blinded method, and the Ki-67 index only evaluated the percentage of positive cells.

\section{Whole-genome sequence}


Genomic DNA was firstly extracted from selected FFPE surgical samples of 9 MEITL cases using QIAamp DNA FFPE tissue kit (Qiagen). To prepare materials for constructing the DNA library, we fragmented $2 \mu \mathrm{g}$ DNA using Covaris M220 sonication system (Covaris), followed by end-repairing, A-tailing, and adaptor ligation and purification by KAPA Hyper Prep Kit (KAPA Biosystems). What's more, the selected DNA fragments needed to be further amplified and purified before the Illumina Rapid Capture Extended Exome Kit (Illumina Inc.) could be used for exome enrichment. Finally, captured libraries were submitted to the Illumina Hiseq 2500 platform (Illumina Inc.) for further sequencing.

Sample quality control criteria: 1 . DNA extracted from each sample $\geq 50$ ng with $A 260 / 280$ ranged from 1.8 to 2.0 ; 2. DNA in the pre-library > 100ng; 3 . Q30 (the percentage of bases with mass value $\geq 30$ ) $>75 \%$; 4. mean sequencing depth of $150 \times$ for tissues and $60 \times$ for negative control tissues.

\section{Sequence analysis and Pathway enrichment analysis}

Paired-end sequencing data after quality control was aligned to the human reference genome hg19 using Burrows-Wheeler Aligner (BWA-MEM) [6], followed by sorting, PCR-duplicate removal, base quality score recalibration(BQSR) using Samtool v1.6, Picard v1.119 (http://picard.sourceforge.net/) and Genome Analysis Toolkit (GAKT)[7], respectively. Single nucleotide variants (SNV) and germline mutations were identified by a useful variant caller - VarDict-1.5.4[8], and Scalpel (http://scalpel.sourceforge.net) was an effective algorithm for mining insertion and deletion mutations[9]. SNVs and indels identified were excluded if present $>1 \%$ frequency in the 1000 Genomic project. The annotations of all SNVs and indels were acquired using vcf2maf-1.6.16. Meanwhile, the CNV kit was performed for analysis of copy number variants (CNV) at the chromosome level.

We performed Gene Ontology (GO, http://www.geneontology.org/) and Kyoto Encyclopedia of Genes and Genomes (KEGG, https://www.kegg.jp/) analysis to explore the biological processes and pathways associated with MEITL.

\section{Prediction of mutations on protein stability}

Polyphen2 (Polymorphism Phenotyping v2), SIFT (Sorting intolerant of tolerant substitution) are used to predict the possible impact of an amino acid substitution on protein structure by generating a score based on each sequence. The default score threshold of SIFT is currently set at 0.05 for binary classification (deleterious: $<0.05$; tolerated: $\geq 0.05$ ) with a balanced accuracy of $76.99 \%$ (http://sift.jcvi.org/)[10]. The score in Polyphen2 ranges from 0 to 1, and the smaller the score, the less likely it is to damage protein stability. According to the score, mutations are divided into benign (0-0.452), possibly damaging (0.447-0.909) and probably damaging (0.909-1) (http://genetics.bwh.harvard.edu/pph2/)[11].

\section{Results}

\section{Clinical findings}


There were 14 men and 6 women with a median age of 62.5 (range, 28-81 years). Among all cases, $17(17 / 20)$ lesions were located in the jejunum or ileum, $2(2 / 20)$ in the colon, and $1(1 / 20)$ in both ileum and colon. Patients had common gastrointestinal symptoms, including anemia (19/20), abdominal pain $(17 / 20)$, and intestinal perforation (10/20). Weight loss (8/20), abdominal mass $(7 / 20)$, fever $(6 / 20)$, bloating $(6 / 20)$, diarrhea $(3 / 20)$, and intestinal obstruction $(3 / 20)$ were observed in less than half of the cases. All patients had no evidence of celiac disease or inflammatory bowel disease.

\section{Pathological features in MEITL}

Seventeen jejunoileal specimens, 1 ileocolic specimen, and 2 colorectal specimens were selected for examination. Grossly, thirteen (13/20) cases showed single/multiple ulcers and perforations, and the mucosal folds around the rupture disappeared, part of the serous membrane had fibrinopurulent exudates. Seven $(7 / 20)$ cases developed solid masses of size ranging from $3.2 \mathrm{~cm} * 2 \mathrm{~cm} * 1.3 \mathrm{~cm}$ to $10 \mathrm{~cm} * 4 \mathrm{~cm} * 1 \mathrm{~cm}$ with grayish-white and reddish cut surface, infiltrating the full- thickness of the intestinal wall.

Microscopically, most tumor cells were monomorphic medium-sized cells with round dark nuclei and pale cytoplasm(Fig. 1A), spreading to adjacent areas and infiltrating the full-thickness of the intestinal wall. Only in one case, the tumor cells were atypical and polymorphic, along with large nuclei, obvious nucleoli, increased mitotic activity and nuclear fragmentation(Fig. 1B), infiltrating the fibrous connective adipose tissue outside the lamina propria, rather than the epithelium and lamina propria. Multinuclear cells and megakaryocytes were also seen. Epitheliotropic pattern(Fig. 1C) was demonstrated in 12(12/20) cases, with obvious neoplastic necrosis at the perforation and scattered acute and chronic inflammatory cells in the interstitium. Villus atrophy, crypt hyperplasia, and increased lymphocytes in the crypt and surface epithelium were found in adjacent mucosae(Fig. 1D). Lymph node involvements were histologically confirmed in $3(3 / 20)$ cases.

Tumor cells in all 20 cases were strongly positive for CD3(Fig. 1E), while CD43(19/20) (Fig. 1F), CD8(17/20) (Fig. 1G), and CD56(15/20) were positive for partial tumor cells. B cell markers, like CD20(5/20) (Fig. 1H) and CD79a(6/20), were positive in scattered tumor cells; whereas CD5 was negative in all cases. The Ki-67 index was generally high ranging from $60-90 \%$. Interestingly, more than half of these cases expressed cytotoxic markers including TIA-1(19/20), Granzyme B(12/20), and perforin(11/20). Moreover, all cases were EBER-ISH negative. (Fig. 1 and Table 1) 
Table 1

The pathological features of 20 MEITL cases.

\begin{tabular}{|ll|}
\hline & $\mathbf{n}(\%)$ (total = 20) \\
\hline Gross & \\
\hline Multiple ulcers & $2(10)$ \\
\hline Perforation & $13(65)$ \\
\hline Mass & $5(25)$ \\
\hline Morphology & \\
\hline Cell morphology & \\
\hline Monomorphic & $19(85)$ \\
\hline Polymorphic & $1(5)$ \\
\hline Infiltration depth & \\
\hline Full-thickness & $18(90)$ \\
\hline Mucosa and submucosa & $2(10)$ \\
\hline Epitheliotropic pattern & \\
\hline Present & $12(60)$ \\
\hline Absent & $8(40)$ \\
\hline Neoplastic necrosis & \\
\hline Present & $20(100)$ \\
\hline Absent & $0(0)$ \\
\hline Mitosis & $11(55)$ \\
\hline Present & \\
\hline Absent & \\
\hline Inflammation & \\
\hline Present & \\
\hline Absent & \\
\hline Lymph node involvements & \\
\hline Present & \\
\hline Absent & \\
\hline
\end{tabular}




\begin{tabular}{|ll|}
\hline & $\mathrm{n}(\%)$ (total = 20) \\
\hline Crypt changes in adjacent mucosae & \\
\hline Present & $20(100)$ \\
\hline Absent & $0(0)$ \\
\hline Immunohistochemistry \\
\hline CD3+ \\
\hline CD5+ \\
\hline CD8+ \\
\hline CD43+ \\
\hline CD56+ & $0(0)$ \\
\hline TIA-1+ & $17(85)$ \\
\hline GranB+ & $19(95)$ \\
\hline perforin+ & $15(75)$ \\
\hline EBER+ & $19(95)$ \\
\hline CD20+ & $12(60)$ \\
\hline CD79a+ & $11(55)$ \\
\hline Ki-67 index & $0(0)$ \\
\hline
\end{tabular}

\section{Molecular genetic findings}

There were 1987 somatic mutations with a median of 220 (range 19-1577) mutations per patient (average of 1.82 per $\mathrm{Mb}$ ), typically consisting of missense mutations(Fig. 2A), and the mutated spectrum showed a predominance of $\mathrm{C}>\mathrm{T}$ and $\mathrm{G}>\mathrm{A}$ transitions(Fig. 2B). $66.7 \%$ of cases had mutations in genes of the JAK-STAT signaling pathway which was known as a major oncogenic mechanism in T cell lymphomas, including STAT5B mutations (4/9), JAK3 mutations (3/9), and STAT5A mutations (2/9) (Fig. 2C). Interestingly, the STAT5A mutation and STAT5B mutation were found to be mutually excluded, although either could active the JAK-STAT pathway. The four STAT5B-mutated cases harbored three distinctive missense variants, including the common N642H mutation(rs938448224) and two mutations -Y665F(COSM1716592) and A766V(COSM8916674) which were only reported in COSMIC(Fig. 3A). TP53 variants were discovered in 4 of 9 cases, and all were located in the DNA-binding domain(Fig. 3B). Two of them were frameshift mutations, V147Afs* 19 and I232Sfs ${ }^{\star} 15$, which were expected to confer critical changes in protein structure, and the other two missense mutations (R248Q and G105C) were predicted to be deleterious with a damaging effect on the protein by SIFT and Polyphen2 algorithms. Four 
of 9 cases harbored ZDBF2 mutations, frameshift mutation K1726Nfs*5(rs745875253) in 3 cases, and missense mutation A564S in 1 case. Only 2 (2/9) cases had SETD2 mutations.

KEGG and GO enrichment analysis revealed that mutated genes expressed higher in MEITL were enriched for the JAK-STAT signaling pathway and DNA damage system(Fig. 2C).

Copy number analysis derived from WGS data identified multiple regions of frequent gains and losses(Fig. 2D). The most significant chromosome copy number variation of MEITL was the amplification of $9 q$, accounting for $77.8 \%$ of all cases. Gains of $7 q(4 / 9), 19 q(3 / 9), 1 q(3 / 9)$ and losses of $7 p(4 / 9), 18 p(3 / 9)$ and $8 p(3 / 9)$ were also frequent in MEITL(Fig. 2D). The changes of gene copy number were consistent with that of chromosome arms. Tumor-associated genes on Chr9q such as FANCC, NTRK2, SYK, and NOTCH1 were amplified; amplification of BRAF, EZH2, MET, CDK6 and HGF located on Chr7 were also detected. Five(5/9) cases had both the amplification of Chr9q and mutations of genes of the JAK-STAT pathway.

Frequent germline mutations were also detected. CADM1 germline variant $c^{\star} 161$ del was documented in 5 out of 9 cases, with the highest frequency, followed by MPP7 C. $952+816 \mathrm{~T}>\mathrm{G}(4 / 9)$, RNF145 c. $-40+$ $1137 A>G(4 / 9)$, PACRG c. $613+6010 T>G(3 / 9)$, CD9 c.538-159C > T(3/9), TMPRSS4 c. ${ }^{*} 169 G>A(3 / 9)$.

\section{Discussion}

The present study analyzed the clinicopathological and molecular genetic characteristics of 20 Chinese patients with MEITL. In line with the previous studies[12, 13], men were more susceptible than women with a ratio of 2:1. MEITL patients commonly displayed primary symptoms of abdominal pain, accompanied by systemic symptoms such as fever, anemia, and weight loss. Complications such as intestinal perforation are also common. MEITL mostly occurred in the small intestine, but could also inflect the whole GI tract[14]. Previous studies reported colon and stomach as primary sites that also involved mesenteric nodules or other distant organs including liver, spleen, lung, bone, or skin[15, 16]. Skin involvement was rare as it only occurred in $5 \%$ of all patients and may present as erythema multiforme skin disease or skin pigmentation[17].

The histological findings and immunohistochemistry of our patients were consistent with those reported in most literature[12,13,18,19], with one exception. The monomorphic and small to medium-sized tumor cells in most cases were positive for CD3, CD8, CD 43, CD56, and negative for CD 5, with round nuclei, obvious nucleoli, and pale cytoplasm. Increased mitotic activity, frequent necrosis, and epitheliotropic pattern could also be seen. While the outlier case showed cellular pleomorphism and lymph node involvement and had a longer overall survival time after CHOP chemotherapy than other patients. We hypothesized that cellular pleomorphism is related to prognosis, but due to the number of our cohort, the possibility of accidental occurrence cannot be ruled out.

WGS results of nine cases showed the genetic landscape of Chinese population. Mutation-induced abnormal activation of the JAK-STAT pathway together with chromatin remodeling and DNA damage 
control pathways were pervasive in diverse $\mathrm{T}$ cell malignancies and were reported to have an important role in oncogenesis by regulating cell proliferation, survival, differentiation, and immune response[20]. As expected, protein-coding genes involved in the JAK-STAT pathway (STAT5B, JAK3, STAT5A), histone modifiers (SETD2, CREBBP), and DNA damage (TP53) were recurrently mutated. The mutation frequencies of these genes, except SETD2, were similar to those in previous reports[3-5, 21]. SETD2 is an important epigenetic gene encoding histone methyltransferase SETD2 protein which is involved in gene transcription extension and mismatch repair, and the inactivation of SETD2 may contribute to cancer development[22]. SETD2 was found to be the most significant recurrently mutated gene and mutated in up to $60 \%$ of patients with MEITL in previous studies reported by Tomita[5], Moffitt[4], and Roberti[3]. But in our study only 2 patients had SETD2 mutations (2/9), showing a lower incidence. Since Tomita's study was based on Asian patients, we ruled out the interference of ethnic heterogeneity, and we guessed it may be caused by the small sample size and environmental factors.

STAT5B was the most frequently mutated gene in our study. As a member of the STAT family, STAT5B plays a key regulatory role in the pathogenesis of various disease drivers, including $B C R / A B L[23]$ and NPM-ALK[24], and expresses at a high level in hematological malignancies[25]. There have revealed multiple mutation hot-spots within SH2 and C-terminal domains in STAT5B, among which $\mathrm{N} 642 \mathrm{H}$ has been discovered in various hematological malignancies of $\mathrm{T}$ cell origin including T-cell acute lymphoblastic leukemia(T-ALL), T-cell prolymphocytic leukemia(T-PLL), and MEITL[26]. Patient with a STAT5B N642H variant has a risk of relapse, drug resistance and poor outcome[27]. $\mathrm{N642H}$ was detected in two of four STAT5B-mutated cases in our study and predicted to be probably damaging by Polyphen-2. Two novel variants $\mathrm{Y} 665 \mathrm{~F}$ and $\mathrm{A} 776 \mathrm{~V}$ had also been found, but needed more research to explore their functions.

Our study showed for the first time frequent ZDBF2 mutations. ZDBF2 is located on chromosome 2q33.3 and encodes a protein containing DBF4-type zinc finger domains, related to nucleic acid binding and zinc ion binding according to GO annotations[28]. So far, only Nasopalpebral Lipoma-Coloboma Syndrome[28] and Temple Syndrome[29], both of which are dominant genetic diseases, have been reported to be associated with ZDBF2. Since frequent frameshift mutation K1726Nfs*5(rs745875253) was discovered in our study and ZDBF2 was reported to be imprinted and paternally expressed in lymphocytes[30], we speculate that ZDBF2 is an important gene contributing to the MEITL mechanism and had been largely overlooked.

Abnormalities of Chr9q were frequently detected in non-Hodgkin lymphoma, especially loss of Chr9q[31]. Both Type-I EATL and MEITL were characterized by Chr9q gains[32], and its frequency could be up to $75 \%$ in MEITL[3, 33]. Our research had verified that amplification of Chr9q(7/9) was the most common chromosome copy number variant in MEITL, and multiple important oncogenes on Chr9q showed consistent amplifications. Five cases also had mutations of JAK-STAT pathway-related genes at the same time. Though recurrent Chr9q gains were reported in mantle cell lymphoma(MCL), its frequency was lower, and the characteristic mutated genes in MCL were not related to the JAK-STAT pathway. These 
findings indicated that a combination of genetic mutations and Chr9q gains could be used as one of the molecular features of MEITL diagnosis.

There were some limitations in the current research. First, due to the small sample size, we have not yet analyzed the correlation between the genetic changes and clinicopathological manifestations. Second, the pathogenic mechanisms of the above genetic changes on MEITL have not been further studied. We will continue to collect Chinese MEITL cases to solve the above problems and verify our results.

\section{Conclusions}

Our work uncovers the clinicopathological and special molecular genetic characteristics of MEITL patients in the Chinese population. MEITL often occurs in elderly males and has non-specific digestive symptoms, with $\mathrm{CD}^{+} / \mathrm{CD}^{+} / \mathrm{CD}^{2} 6^{+} / \mathrm{CD}^{-}$monomorphic medium-sized tumor cells and obvious epitheliotropic performance. Frequent mutations of TP53, ZDBF2, and genes affecting the JAK-STAT pathway, especially STAT5B, are common to be discovered. Combining the mutant genes involved in the JAK-STAT pathway and the amplification of Chr9q may be a powerful auxiliary tool for diagnosing MEITL.

\section{Abbreviations}

MEITL Monomorphic Epitheliotropic Intestinal T-cell Lymphoma

IHC Immunohistochemistry

WGS Whole-genome sequence

SNV single nucleotide variant

CNV copy number variant

GO Gene Ontology

KEGG Kyoto Encyclopedia of Genes and Genomes

MCL mantle cell lymphoma

\section{Declarations}

\section{Ethics approval}

The use of samples in this study was in accordance with the Declaration of Helsinki and approved by the Ethics Committee of The First Affiliated Hospital of Nanjing Medical University (2020-SR-169). 
Not applicable.

\section{Availability of data and materials}

The datasets generated and/or analyzed during the current study are not publicly available but are available from the corresponding author on reasonable request.

\section{Competing interests}

The authors declare that they have no competing interests.

\section{Source of Funding}

This work was supported by the National Natural Science Foundation of China (81773109), the Natural Science Foundation of Jiangsu Province (BK20151582), the National key Clinical Specialty Construction Project (2014), Joint key project funded by Southeast University and Nanjing Medical University (2242019K3DN09, JX218GSP20190735) and the Fund of the priority Academic Program Development of Jiangsu Higher Education Institution (JX1023-1801).

\section{Authors' contributions}

Z. Zhang designed research; C. Chen, Y. Gong, Q. Xia, Q. Rao, X. Zhang collected data; C. Chen, Y. Gong, J. Zhang and B. Zhai analyzed data; Y. Shao, L. Zhu, X. Li and P. Ji conducted the experiments; C. Chen, Y. Gong and Y. Yang wrote the paper; Z. Zhang supported administratively; Z. Zhang revised the paper. All authors contributed to and have approved the final manuscript.

\section{Acknowledgements}

Not applicable.

\section{References}

1. Swerdlow SH, Campo E, Pileri SA, Harris NL, Stein H, Siebert R, et al. The 2016 revision of the world health organization classification of lymphoid neoplasms. Blood. 2016;127(20):2375-90. doi:10.1182/blood-2016-01-643569.

2. Tse E, Gill H, Loong F, Kim SJ, Ng SB, Tang T, et al. Type ii enteropathy-associated t-cell lymphoma: a multicenter analysis from the asia lymphoma study group. Am J Hematol. 2012;87(7):663-8. doi:10.1002/ajh.23213.

3. Roberti A, Dobay MP, Bisig B, Vallois D, Boechat C, Lanitis E, et al. Type ii enteropathy-associated tcell lymphoma features a unique genomic profile with highly recurrent setd2 alterations. Nat Commun. 2016;7:12602. doi:10.1038/ncomms12602.

4. Moffitt AB, Ondrejka SL, Mckinney M, Rempel RE, Goodlad JR, Teh CH, et al. Enteropathy-associated t cell lymphoma subtypes are characterized by loss of function of setd2. J Exp Med. 
2017;214(5):1371-86. doi:10.1084/jem.20160894.

5. Tomita S, Kikuti YY, Carreras J, Sakai R, Takata K, Yoshino T, et al. Monomorphic epitheliotropic intestinal t-cell lymphoma in asia frequently shows setd2 alterations. Cancers (Basel). 2020;12(12). doi:10.3390/cancers12123539.

6. Li H, Durbin R. Fast and accurate long-read alignment with burrows-wheeler transform. Bioinformatics. 2010;26(5):589-95. doi:10.1093/bioinformatics/btp698.

7. Mckenna A, Hanna M, Banks E, Sivachenko A, Cibulskis K, Kernytsky A, et al. The genome analysis toolkit: a mapreduce framework for analyzing next-generation dna sequencing data. Genome Res. 2010;20(9):1297-303. doi:10.1101/gr.107524.110.

8. Lai Z, Markovets A, Ahdesmaki M, Chapman B, Hofmann O, Mcewen R, et al. Vardict: a novel and versatile variant caller for next-generation sequencing in cancer research. Nucleic Acids Res. 2016;44(11):e108. doi:10.1093/nar/gkw227.

9. Fang H, Bergmann EA, Arora K, Vacic V, Zody MC, lossifov I, et al. Indel variant analysis of short-read sequencing data with scalpel. Nat Protoc. 2016;11(12):2529-48. doi:10.1038/nprot.2016.150.

10. Choi Y, Sims GE, Murphy S, Miller JR, Chan AP. Predicting the functional effect of amino acid substitutions and indels. Plos One. 2012;7(10):e46688. doi:10.1371/journal.pone.0046688.

11. Adzhubei IA, Schmidt S, Peshkin L, Ramensky VE, Gerasimova A, Bork P, et al. A method and server for predicting damaging missense mutations. Nat Methods. 2010;7(4):248-9. doi:10.1038/nmeth0410-248.

12. Yi JH, Lee GW, Do YR, Jung HR, Hong JY, Yoon DH, et al. Multicenter retrospective analysis of the clinicopathologic features of monomorphic epitheliotropic intestinal t-cell lymphoma. Ann Hematol. 2019;98(11):2541-50. doi:10.1007/s00277-019-03791-y.

13. Fei F, Reddy V, Patel CR, Dhall D, Lee G, Meng-Jun X, et al. Monomorphic epitheliotropic intestinal tcell lymphoma: a study of four cases and review of literature. Ann Clin Lab Sci. 2020;50(6):806-12.

14. Delabie J, Holte H, Vose JM, Ullrich F, Jaffe ES, Savage KJ, et al. Enteropathy-associated t-cell lymphoma: clinical and histological findings from the international peripheral t-cell lymphoma project. Blood. 2011;118(1):148-55. doi:10.1182/blood-2011-02-335216.

15. Deleeuw RJ, Zettl A, Klinker E, Haralambieva E, Trottier M, Chari R, et al. Whole-genome analysis and hla genotyping of enteropathy-type t-cell lymphoma reveals 2 distinct lymphoma subtypes. Gastroenterology. 2007;132(5):1902-11. doi:10.1053/j.gastro.2007.03.036.

16. Gale J, Simmonds PD, Mead GM, Sweetenham JW, Wright DH. Enteropathy-type intestinal t-cell lymphoma: clinical features and treatment of 31 patients in a single center. $\mathrm{J}$ Clin Oncol. 2000;18(4):795-803. doi:10.1200/JC0.2000.18.4.795.

17. Aiempanakit K, Amatawet C, Chiratikarnwong K, Auepemkiate S, Kayasut K, Suwiwat S, et al. Erythema multiforme-like cutaneous lesions in monomorphic epitheliotropic intestinal t-cell lymphoma: a rare case report. J Cutan Pathol. 2017;44(2):183-8. doi:10.1111/cup.12864.

18. Tan SY, Chuang SS, Tang T, Tan L, Ko YH, Chuah KL, et al. Type ii eatl (epitheliotropic intestinal t-cell lymphoma): a neoplasm of intra-epithelial t-cells with predominant cd8alphaalpha phenotype. 
Leukemia. 2013;27(8):1688-96. doi:10.1038/leu.2013.41.

19. Chan JK, Chan AC, Cheuk W, Wan SK, Lee WK, Lui YH, et al. Type ii enteropathy-associated t-cell lymphoma: a distinct aggressive lymphoma with frequent gammadelta t-cell receptor expression. Am J Surg Pathol. 2011;35(10):1557-69. doi:10.1097/PAS.0b013e318222dfcd.

20. Orlova A, Wingelhofer B, Neubauer HA, Maurer B, Berger-Becvar A, Keseru GM, et al. Emerging therapeutic targets in myeloproliferative neoplasms and peripheral t-cell leukemia and lymphomas. Expert Opin Ther Targets. 2018;22(1):45-57. doi:10.1080/14728222.2018.1406924.

21. Nairismagi ML, Tan J, Lim JQ, Nagarajan S, Ng CC, Rajasegaran V, et al. Jak-stat and g-proteincoupled receptor signaling pathways are frequently altered in epitheliotropic intestinal t-cell lymphoma. Leukemia. 2016;30(6):1311-9. doi:10.1038/leu.2016.13.

22. Li J, Duns G, Westers H, Sijmons R, van den Berg A, Kok K. Setd2: an epigenetic modifier with tumor suppressor functionality. Oncotarget. 2016;7(31):50719-34. doi:10.18632/oncotarget.9368.

23. Kollmann S, Grundschober E, Maurer B, Warsch W, Grausenburger R, Edlinger L, et al. Twins with different personalities: stat5b-but not stat5a-has a key role in bcr/abl-induced leukemia. Leukemia. 2019;33(7):1583-97. doi:10.1038/s41375-018-0369-5.

24. Nieborowska-Skorska M, Slupianek A, Xue L, Zhang Q, Raghunath PN, Hoser G, et al. Role of signal transducer and activator of transcription 5 in nucleophosmin/ anaplastic lymphoma kinasemediated malignant transformation of lymphoid cells. Cancer Res. 2001;61(17):6517-23.

25. Stark GR, Darnell JJ. The jak-stat pathway at twenty. Immunity. 2012;36(4):503-14. doi:10.1016/j.immuni.2012.03.013.

26. de Araujo ED, Erdogan F, Neubauer HA, Meneksedag-Erol D, Manaswiyoungkul P, Eram MS, et al. Structural and functional consequences of the stat5b(n642h) driver mutation. Nat Commun. 2019;10(1):2517. doi:10.1038/s41467-019-10422-7.

27. Waldmann TA, Chen J. Disorders of the jak/stat pathway in t cell lymphoma pathogenesis: implications for immunotherapy. Annu Rev Immunol. 2017;35:533-50. doi:10.1146/annurevimmunol-110416-120628.

28. Chacon-Camacho OF, Sobreira N, You J, Pina-Aguilar RE, Villegas-Ruiz V, Zenteno JC. Exome sequencing identifies a de novo frameshift mutation in the imprinted gene zdbf2 in a sporadic patient with nasopalpebral lipoma-coloboma syndrome. Am J Med Genet A. 2016;170(7):1934-7. doi:10.1002/ajmg.a.37683.

29. Kagami M, Matsubara K, Nakabayashi K, Nakamura A, Sano S, Okamura K, et al. Genome-wide multilocus imprinting disturbance analysis in temple syndrome and kagami-ogata syndrome. Genet Med. 2017;19(4):476-82. doi:10.1038/gim.2016.123.

30. Kobayashi H, Yamada K, Morita S, Hiura H, Fukuda A, Kagami M, et al. Identification of the mouse paternally expressed imprinted gene zdbf2 on chromosome 1 and its imprinted human homolog zdbf2 on chromosome 2. Genomics. 2009;93(5):461-72. doi:10.1016/j.ygeno.2008.12.012.

31. Offit K, Parsa NZ, Jhanwar SC, Filippa D, Wachtel M, Chaganti RS. Clusters of chromosome 9 aberrations are associated with clinico-pathologic subsets of non-hodgkin's lymphoma. Genes 
Chromosomes Cancer. 1993;7(1):1-7. doi:10.1002/gcc.2870070102.

32. Zettl A, Ott G, Makulik A, Katzenberger T, Starostik P, Eichler T, et al. Chromosomal gains at 9q characterize enteropathy-type t-cell lymphoma. Am J Pathol. 2002;161(5):1635-45. doi:10.1016/S0002-9440(10)64441-0.

33. Tomita S, Kikuti YY, Carreras J, Kojima M, Ando K, Takasaki H, et al. Genomic and immunohistochemical profiles of enteropathy-associated t-cell lymphoma in japan. Mod Pathol. 2015;28(10):1286-96. doi:10.1038/modpathol.2015.85.

\section{Figures}



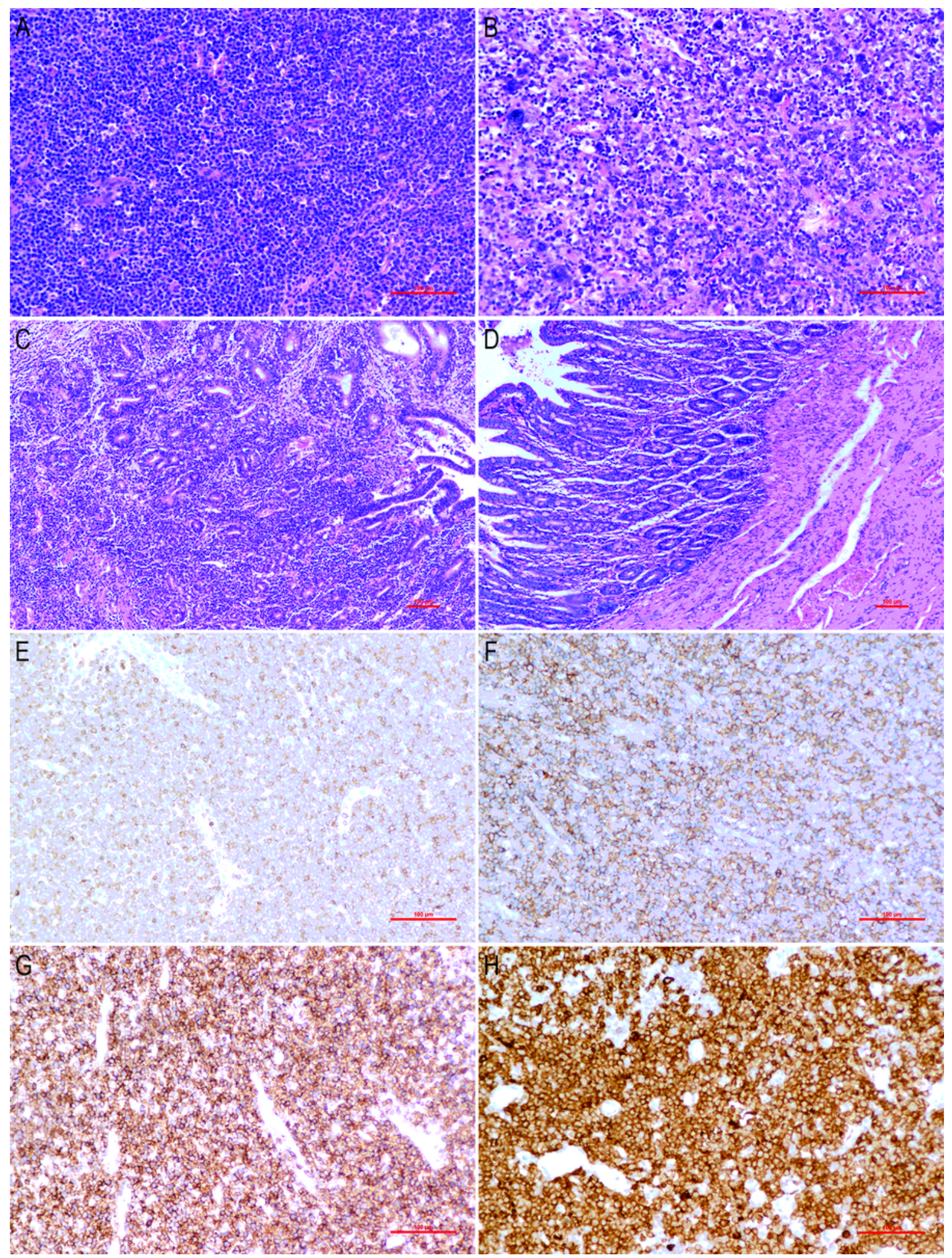

\section{Figure 1}

Monomorphic epitheliotropic intestinal T囚cell lymphoma A-H: ×10 (A) monomorphic medium-sized tumor cells with round dark nuclei and pale cytoplasm; (B) atypical and polymorphic tumor cells, along with large nuclei, obvious nucleoli, increased mitotic activity and nuclear fragmentation; (C) epitheliotropic pattern; (D) villus atrophy, crypt hyperplasia, and increased lymphocytes in the crypt and surface 
epithelium; (E) CD3+; (F) CD43+; (G) CD8+; (H) CD20+. (A-D: hematoxylin and eosin staining, E-H: IHC staining)
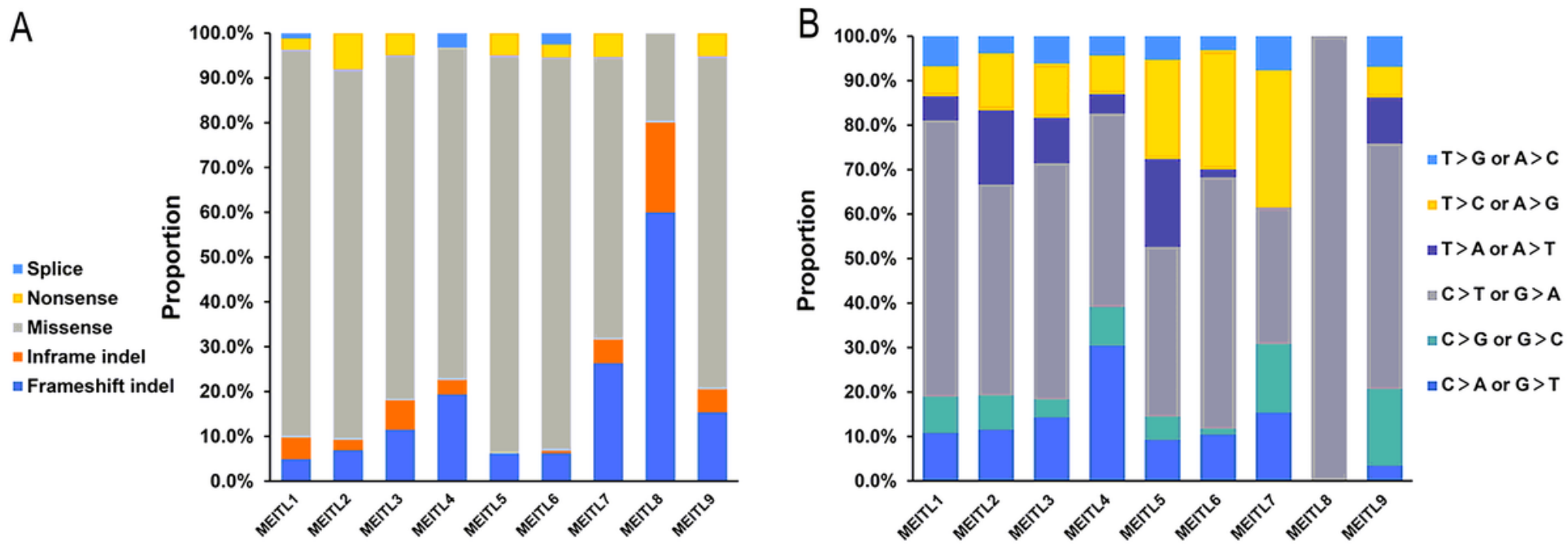

C

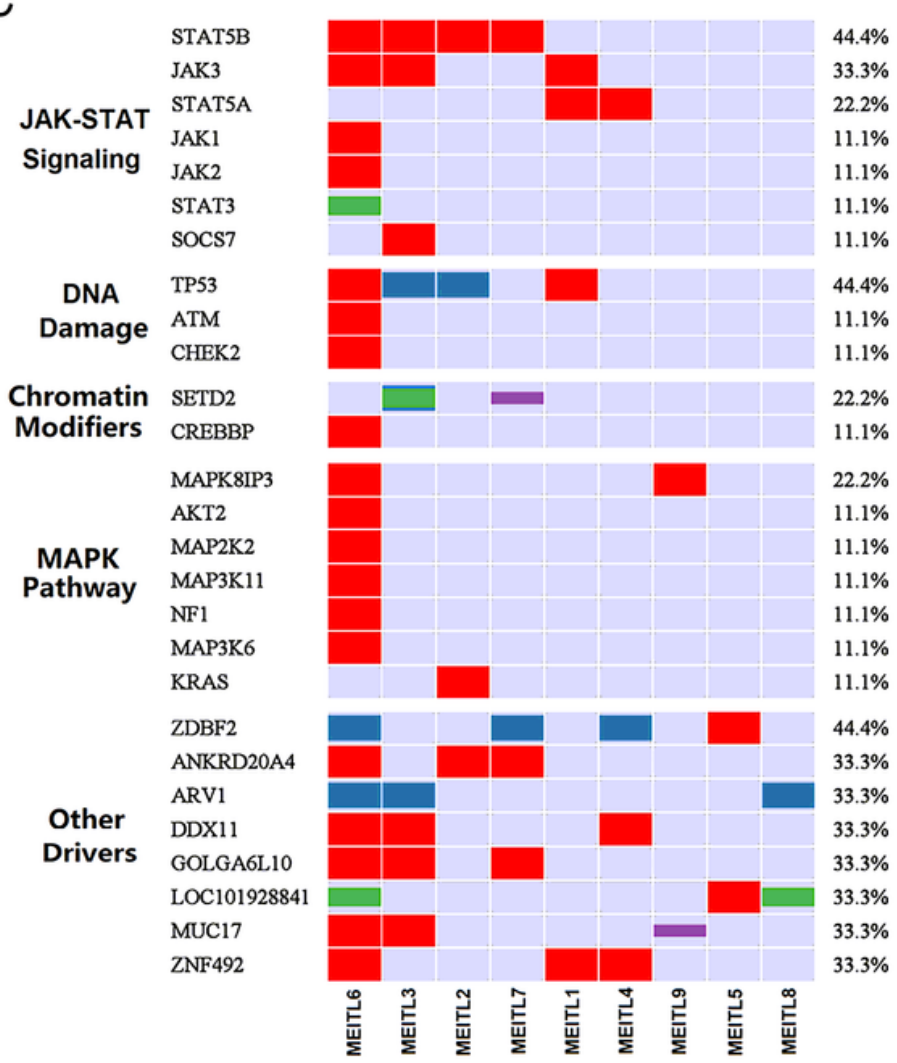

D

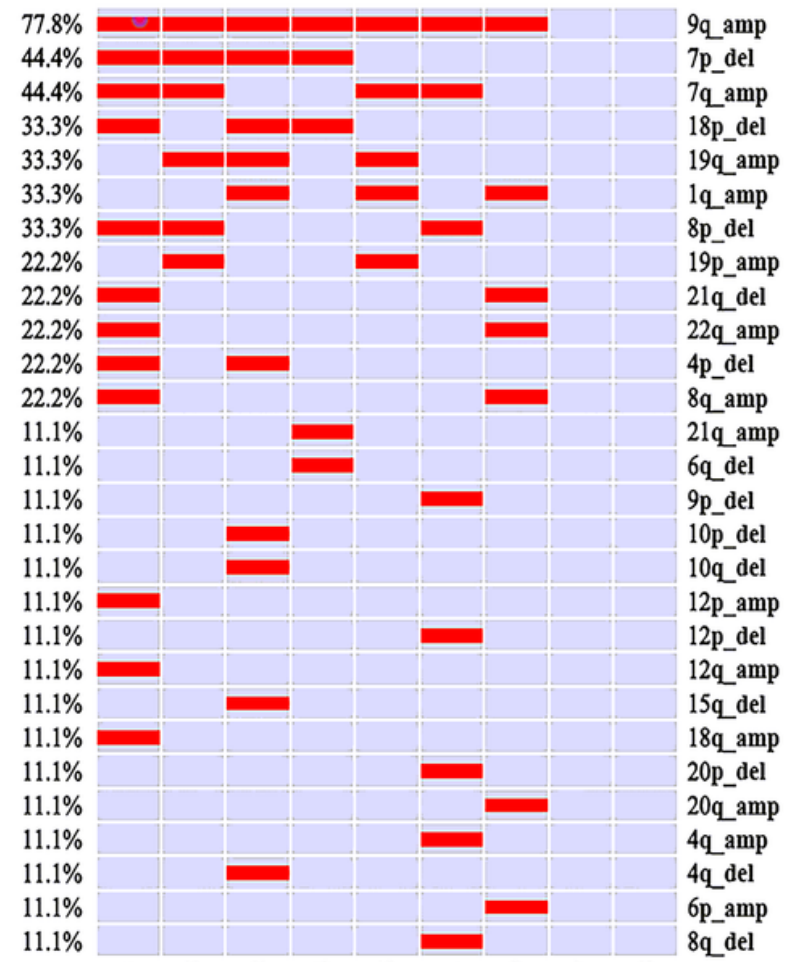

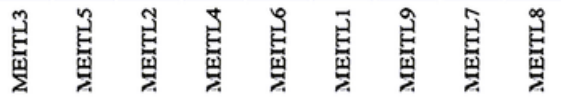

\section{Figure 2}

MEITL mainly consisted of missense mutations $(A)$ and had a predominance of $C>T$ and $G>A$ transitions (B); (C) depicted the heat map and associated signal pathways of MEITL, and $66.7 \%$ of cases had mutations in genes of JAK-STAT signaling pathway, including STAT5B, JAK3 and STAT5A; (D) showed the major copy number variants in MEITL, and the most significant chromosome copy number variation was the amplification of $9 q$, followed by gains of $7 q$ and losses of $7 p$. 
A

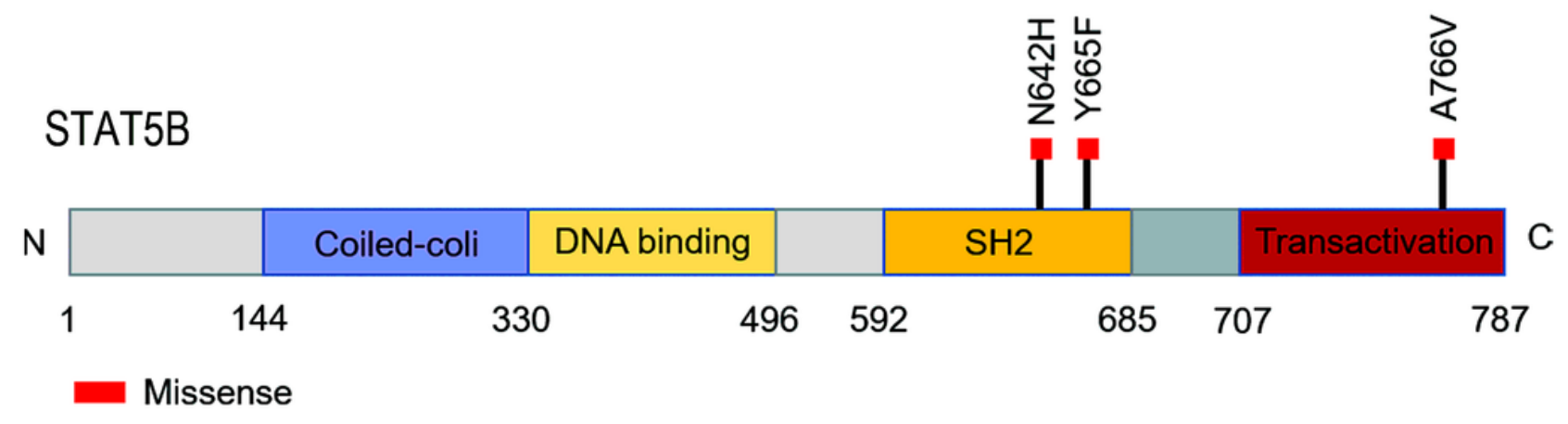

B

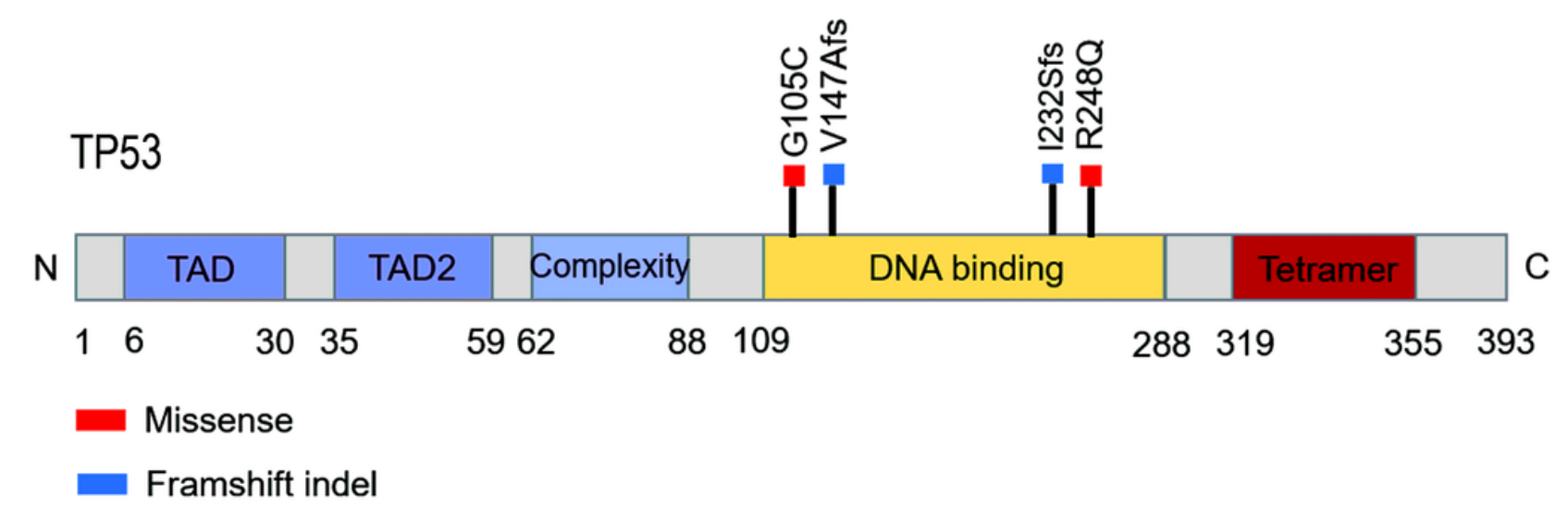

Figure 3

Somatic alterations in STAT5B and TP53 identified in this study. (A) Four STAT5B-mututed cases harbored three distinctive missense variants, including the common N642H mutation(rs938448224) and two mutations-Y665F(COSM1716592) and A766V(COSM8916674); (B) Four TP53-mututed cases harbored two frameshift variants - V147Afs*19 and I232Sfs, and two missense variants - R248Q and G105C. 